\title{
Efficiency and mechanism of dust removal with charged water mist in vibration fiber grid
}

\author{
Chen Zu-yun ${ }^{\mathrm{a}, \mathrm{b}, *}$, Peng Ji-cheng ${ }^{\mathrm{a}, \mathrm{b}}$, Rao Zhen-biao ${ }^{\mathrm{a}, \mathrm{b}}$ \\ a School of Resource and Environmental Engineering, Jiangxi University of Science and Technology, \\ Ganzhou 341000 China \\ b Jiangxi Key Laboratory of Mining \& Metallurgy Environmental Pollution Control, Ganzhou 341000 \\ China \\ *Corresponding author, e-mail: welczy@163.com
}

Received 19 Sep 2020

Accepted 13 Jul 2021

\begin{abstract}
With the salience of air pollution problems such as PM2.5, the removal of micro particle dust has increasingly become a widespread issue to address. In this study, we put forward the technology of charged water mist in vibrating fiber grid. Five types of effect of catching dust, such as gravitational settlement, inertial collision, interception, diffusion, and electrostatic attraction in a single fiber, were theoretically analyzed. The mechanism of vibration fiber grid, whose resonant film-forming effect was analyzed on spray charging water, was also studied. The infinitesimal analysis method was used for calculating the efficiency of catching dust in the vibrating fiber grid. The fiber grating arrangement was determined by performing lots of experiments of dust collection efficiency and resistance loss in longitude of fiber grating, latitude, and interlacing fiber grating. The obtained results showed that not only the dust removal in longitude of fiber grating, which was easy to use, was stable and efficient, but also the resistance loss was relatively low and the natural dust drop did not need frequent ash removal. Therefore, the charged spray in longitude of vibration fiber grating has certain application value in dust removal technology. The theoretical and experimental results also show that the technology of dust removal by charged spray in vibration fiber grid has a high purification efficiency for micro particles and simple structure and thus would have certain valuable application in dust removal technology in future.
\end{abstract}

KEYWORDS: dust collector, dust removal efficiency, vibration fiber grid, charged water mist

\section{INTRODUCTION}

Dust is very harmful to the environment and human body, and controlling dust in the atmosphere is very necessary. Studies have shown that the nonwater-filled vibrating string filter is not effective for removing fine dust. Dust removal by charged water mist mainly relies on the electrostatic gravitational force (Coulomb force) between the charged droplet and fine particles for capture, which in turn weakens the dependence of fine particles on their own inertia and reduces the particle flow with the mobility, improving the effect of fine particle capture. However, the vibratory fiber grid with two pieces has the characteristics of high dust removal efficiency, simple structure, and low cost, and has important social significance for environmental protection, resource recovery, and effective collection of fine dust. It is a very complicated process to catch dust by electrically charged mist in vibration fiber grid, and the charged dust mist is combined with various effects $[1,2]$. Many scholars concluded that the unit vibration fiber to trap dust mainly depends on the combination effects of interception, which play the role of inertia collision and diffusion [3-5]. When water is continuously blown onto the fibers, a water film will be formed on the fibers. The thickness of the water film increases the diameter of the fibers, thereby improving the efficiency of trapping dust through the filtration process [6-8]. The high frequency vibration, which is produced in the vibrating string grid under the action of highspeed airflow, greatly increases the collision probability between dust, droplets, and strings, thus effectively improving the dust removal efficiency. At the same time, under the action of wind, when the moist textile fiber has resonated, the water membrane between the fibers is produced twice [9-12]. The factors affecting the dust removal efficiency were analyzed for investigating the dust removal mechanism of wet vibrating string grid, indicating that the dust removal efficiency by vibrating string 
grid is related to material, vibration frequency, overcurrent section area, layer number, and spray parameters of the vibrating string grid. Its positive role lies in increasing the watering of the active surface area, which in turn improves the probability to catch dust and is also the factor to enhance the dust removal efficiency [13-16]. Moreover, the mechanism of vibration fiber grid was theoretically investigated. Some factors affecting the response amplitude of resonance were investigated. The resonant amplitude is directly related to the external factors of fiber grating structure and material, and the efficiency calculation of the concentrated string fiber grid was obtained by the infinitesimal analysis method. The experimental results indicated that the use of charged water mist combined with the fiber grid technology significantly improved the removal of fine dust. Especially, at small wind speed, the resistance loss of the charged water mist fiber grid would be very small and the efficiency of dust capture would be high, reaching more than $90 \%$. This study can provide a reference for the research of the combination of charged water mist and dust removal technology by fiber grid. Therefore, investigating the dust removal mechanism and experiment of vibrating fiber grid with charged mist is of great significance to control the industrial smoke pollution by the fine dust removal technology.

\section{MATERIALS AND METHODS}

\section{Analysis of mechanism of dust removal by single vibrating fiber}

Dust catching by single vibration string mainly depends on gravity subsidence, inertia collision, interception, diffusion, and electrostatic attraction. According to the principle of fluid mechanics $[17,18]$, the mechanism of dust removal was analyzed theoretically in single fiber filament and dense fiber grating, and the calculation formulas were deduced in fiber grating resistance and local resistance. By comparing the mass flow rate of dust water mist and the accumulation state of dust water droplets on the fiber grating, both the settlement rules and accumulation modes were investigated for different materials and clearance fiber gratings, and the characteristics of dust removal were studied for fiber gratings of different materials with water mist and charging water mist in the experiments.

\section{Effect of gravity subsidence}

The charged droplet of containing dust undergoes a parabolic motion, under its resulting force of gravity and fluid force. When the droplets reach the filament, the dust settles along the filament and is removed with gravity as the main force. In the dust collector, the efficiency of dust removal increases with decreasing inlet wind speed. When the fluid in the dust removing case flows horizontally, the gravity sedimentation efficiency $\eta_{G}$ is calculated by the following formula [19].

$$
\eta_{G}=\frac{G}{\sqrt{1+G^{2}}}, \quad \text { where } \quad G=\frac{\rho_{w} g d_{w}^{2}}{18 \mu \nu},
$$

$\rho_{w}$ is the droplet density, $\mathrm{kg} / \mathrm{m}^{3} ; g$ is the acceleration of gravity, $g=9.8 \mathrm{~m} / \mathrm{s}^{2} ; \mu=$ is the air resistance coefficient, at normal temperature $\mu=$ $1.8 \times 10^{-5} \mathrm{pa} \cdot \mathrm{s} ; v$ is the average inflow velocity of fiber grating, $\mathrm{m} / \mathrm{s}$; and $d_{w}$ is the droplet equivalent diameter, $\mathrm{m}$.

\section{Inertial collision effect}

In the case of the dust droplets with larger diameter than $2.5 \mu \mathrm{m}$, the inertial impact of dust droplets is dominant effect. The fiber has the vibration displacement under the function of air current. Due to the flow characteristics of the fluid around the fiber, curved surface fluid can be formed in a certain area around the fiber. According to the offset range of filaments, the formation of the dynamic surface is also limited within a certain range [20]. When the big mass and volume of droplets contain dust along with the horizontal flow, the dust arrives in this curved surface fluid scope of fibers, because of inertia. The droplets do not immediately flow with the surface fluid, and its trajectory will shift relative to its inertial size. After the migration, the dust droplets are placed in the amplitude range of the vibration string fiber, and some of the droplets collide with the filaments of the fiber, and are eventually captured [19].

The inertia size of the dust droplets is the determining factor in the effect of inertia impact, and their inertia size can be expressed by (Stokes number), as follows:

$$
S_{\mathrm{tk}}=\frac{\rho_{w} d_{w}^{2} v}{9 \mu D} .
$$

In the calculation of efficiency of dust collection, when the Reynolds number $\operatorname{Re} \leqslant 10$, the empirical formula of the efficiency of inertial impact by vibrating fiber was proposed by Landahl and Herman:

$$
\eta_{I}=\frac{S_{\mathrm{tk}}^{3}}{S_{\mathrm{tk}}^{3}+0.77 S_{\mathrm{tk}}^{2}+0.22} .
$$


When Reynolds number Re was greater 10, the empirical formula of the efficiency of inertial impact by vibrating fiber was adopted by Subramanyam as follows:

$$
\eta_{I}=\frac{S_{\mathrm{tk}}}{S_{\mathrm{tk}}+1.5} .
$$

The wind speed $v$ in this experiment was approximately in the range $1-16 \mathrm{~m} / \mathrm{s}$, and the Re was approximately in the range 20-320, when the relevant parameters were taken into account. Because of $\operatorname{Re}>10$, the wind speed could be approximated as a potential flow state, and its capture efficiency of inertial impact could be calculated by formula (4).

\section{Interception effect}

The droplets in the vibrating fiber are affected by the combined action of both the effect of gravity sedimentation and inertial collision. The influence of these two factors on the dust collection is either a large droplet mass or a low flow velocity [15]. When droplet containing dust mass is small or its velocity is slow, its gravity and inertia effects are very small. At this time, they can be approximated to only volume without quality of particles. Then, the droplets move under the flow of circumferential movement fluid [20]. The interception effect of the fiber is known as the interception of the filament when the droplets move along the curved surface of the vibration fiber.

According to the experimental conditions, the potential and flow functions in the potential flow state are expressed by Eqs. (5) and (6), respectively [21]:

$$
\begin{aligned}
& \varphi=v\left(r+\frac{d^{2}}{r}\right) \cos \theta, \\
& \psi=v\left(r-\frac{d^{2}}{r}\right) \sin \theta,
\end{aligned}
$$

where $d$ is the fiber radius, $\mathrm{m} ; r$ is the polar radius, $\mathrm{m}$; and $\theta$ is the polar coordinate angle.

At that time, when $\theta=\pi / 2$ and $r=d+d_{w} / 2$, the streamline equation is obtained by substituting Eq. (6) as follows:

$$
\begin{aligned}
\psi=v(1- & \left.\left(\frac{d}{r}\right)^{2}\right) r \sin \theta \\
& =v\left(1-\left(\frac{d}{d+d_{w} / 2}\right)^{2}\right)\left(d+\frac{d_{w}}{2}\right) .
\end{aligned}
$$

When $r \rightarrow \infty$, then $r=b / \sin \theta$, and the efficiency of dust collection can be obtained by substituting Eq. (7) based on the effect of fiber interception as follows:

$$
\eta_{R}=\frac{b}{d}=1+\frac{d_{w}}{2 d}-\frac{1}{1+d_{w} / 2 d}=\frac{R^{2}+2 R}{1+R},
$$

where $b$ is the distance between limit streamlined and filamentary horizontal axis, $\mathrm{m}$; $R$ is the intercept coefficient; $R=d_{w} / D$, $\mathrm{m} ; d_{w}$ is the droplet equivalent diameter, $\mathrm{m}$; $D$ is the dust particle diameter, $\mathrm{m}$.

Because $0 \leqslant \eta_{R} \leqslant 1$, this can result in $0 \leqslant d_{w} \leqslant$ $\frac{\sqrt{5}-1}{2} D$; therefore, the interception effect can be calculated by Eq. (8) when the unequal relationship is satisfied with $d_{w}$ and $D$, where $d_{w}$ and $D$ are the droplet equivalent diameter and the dust particle diameter, respectively.

\section{Diffusion effect}

The fine dusts, dust droplets, and fog droplets, with particles diameter less than $10 \mu \mathrm{m}$, follow the Brown movement in the dust collector. These particles do not vary with body fluid flow in movement, but the intensity of case particles in the Brownian motion is affected by temperature, pressure, humidity, and other factors. Therefore, the efficiency of diffusion capture is related to the Reynolds number and the particle diffusion ratio in the dust collector, and the diffusion ratio of particle can be calculated by Pe (Bakelite number) [19-22]:

$$
\mathrm{Pe}=\frac{v d_{w}}{S}, \quad \text { where } S=\frac{k_{B} T C_{u}}{3 \pi \mu d_{w}} .
$$

Pe is the particle diffusion ratio; $S$ is the coefficient of diffusion, $\mathrm{m}^{2} / \mathrm{s} ; k_{B}$ is the Boltzmann constant, $k_{B}=1.38 \times 10^{-23} \mathrm{~J} / \mathrm{K} ; T$ is the absolute temperature, $K$; and $C_{u}$ is the Cunningham coefficient of correction,

$$
C_{u}=1+\frac{2 \lambda}{d_{w}}\left(1.257+0.4 \exp \left(-1.1 \frac{d_{w}}{2 \lambda}\right)\right)
$$

where $\lambda$ is the average free path of air molecules.

For the potential flow state of $\operatorname{Re}>1$ and $\mathrm{Pe}>$ 1 , the empirical formula can be used to calculate the trapping efficiency of diffusion effects and is expressed as

$$
\eta_{S}=\sqrt{\frac{8}{\mathrm{Pe}}}
$$

By calculation, when the diameter of dust particle $D$ is less than $0.1 \mu \mathrm{m}$ ( $D$ is the diameter of dust particle), the efficiency of diffusion capture is $80 \%$; when the diameter of dust particle is in the range $0.1 \leqslant D \leqslant 1 \mu \mathrm{m}$, the diffusion effect cannot be 
ignored; when $D>1 \mu \mathrm{m}$, the diffusion effect is negligible. The water droplets are hyperbaric spraying and corona discharge in this experiment, charged droplets and part of dust particles, with a wide range of particle size distribution, and diffusion trapping effect should not be neglected in this test.

\section{Effect of electrostatic attraction}

In this experiment, corona charging dusts were obtained by negative high-voltage power. In the process of applying high negative voltage to the corona, the electric corona will start to aureole with increasing voltage goes up to a certain value. Then, the air ionization around the corona ring has a lot of positive negative ions. Droplet dusts are charged by diffusion charging and electric field charging. A high voltage electric field is formed between the corona pole and the vibrating fiber grid; the charged droplets and dusts are driven into the vibration fiber grid under the electric field force, with high trapping efficiency.

The equivalent formula for electrostatic trapping efficiency is proposed in combination with the charge efficiency and the efficiency of the fiber wire to intercept charged particles, as follows [23]:

$$
\eta_{E}=\eta_{V}+\frac{E q_{f} C_{u}}{3 \pi \mu d_{w} v}\left(\frac{C_{D}}{1+N_{R}}+1+N_{R}\right)
$$

where $\eta_{V}$ is the interception efficiency in potential flow, $\eta_{V}=\left(1+N_{R}\right)-1 /\left(1+N_{R}\right)$; and $C_{D}$ is the filament polarization.

\section{Experiments on vibration fiber grating Vibration fiber grating}

The vibration fiber grid is mainly composed of two parts: the fixed frame and fibers. Both the upper and the lower of the frame are metal screw with a diameter of $10 \mathrm{~mm}$, a stainless steel sheet with a thickness of $2 \mathrm{~mm}$ on the left and right, and a fiber grating as $33 \times 34.5 \mathrm{~cm}^{2}$ frame, in which the fiber grating has an area of $31 \times 31.5 \mathrm{~cm}^{2}$. In order to fix the frame and adjust the fiber simultaneously as vertically as possible, two nuts are installed on each of the four corners of the frame.

There are four types of materials: nylon, plastic, polyester, and stainless steel wire, in which the fiber diameter $D_{f}$ is $0.3 \mathrm{~mm}$, and the gap between the fibers is $0.6 \mathrm{~mm}$. These four materials were used to make vibration fiber grating.

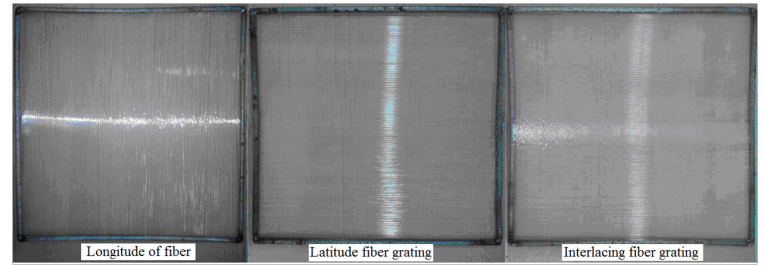

Fig. 1 Fiber grating with different fiber arrangements.
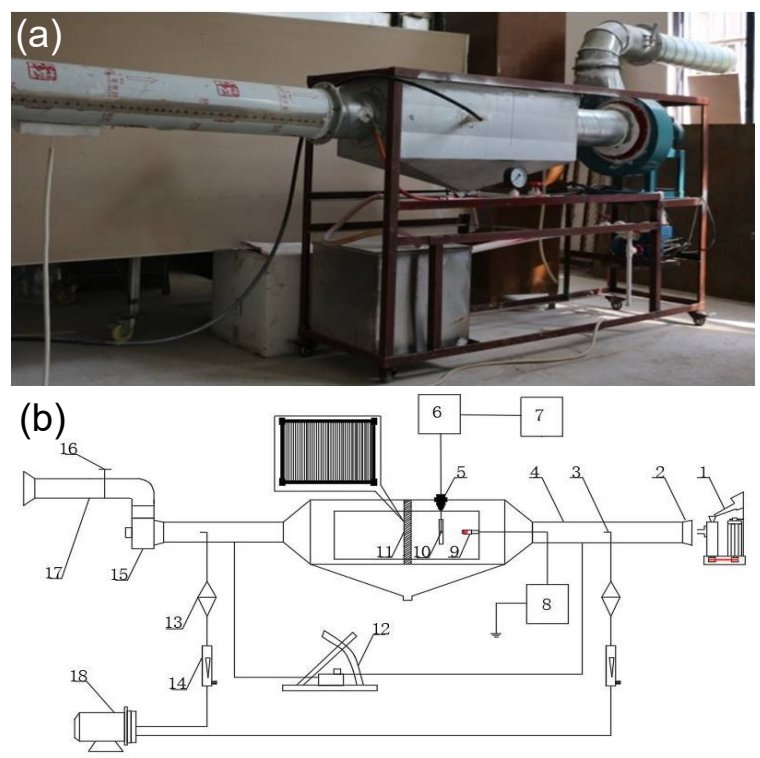

Fig. 2 (a) Experimental device and (b) schematic diagram of all components in the system: 1 , dust generator; 2 , current collection hood; 3 , sampling head; 4, inletpipe; 5 , high voltage insulator; 6 , high voltage source; 7 , control cabinet; 8, pressurized water mist system; 9 , spray nozzle; 10, ring thorn electrode; 11, vibrating string grid; 12 , tilt differential pressure gauge; 13, filter box; 14, rotary flowmeter; 15 , centrifugal fan; 16 , air volume control valve; 17, exhaust duct; and 18, air extracting pump.

\section{Three kinds of fiber grating with longitude, latitude, and interlacing arrangement}

In theory, the filament arranged to longitudinal direction of fiber grating in the fiber grid is more conducive to trap and settle dust droplets. There are three types of fiber grating with longitude, latitude, and interlacing arrangement, as shown in Fig. 1. In order to confirm the optimum layout, verification experiments were carried out before the equipment is manufactured. 


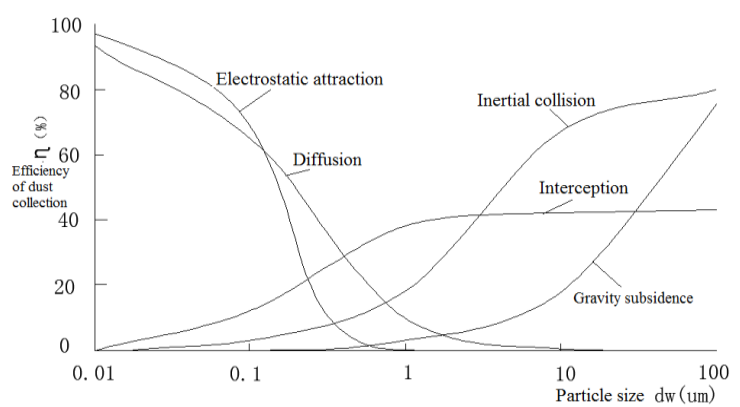

Fig. 3 Efficiency of dust collection of varying particle size by various trapping effect.

\section{Experimental device and diagram}

The model of the spray charged was made and adopted by the dust collector in vibrating string grating in this experiment, and its schematic diagram is shown in Fig. 2. This model mainly comprises six parts of sending dust, ventilation system, water supply system, power supply system, spray charging system, and dust collector system in the vibrating fiber grid. The experimental dust was 270 mesh limestone powder treated by drying through the oven, and the dust emission rate was changed by adjusting the inclination angle of the dust guiding tube in the dust generator.

\section{RESULTS}

Efficiency of dust collection by single string fiber

The analysis and calculation of the efficiency of dust collection by various trapping effects of single vibrating string fiber led to the rule, as shown in Fig. 1. In the case of certain flow velocity, when the particle size of dust droplets $d_{w}$ was greater than $40 \mu \mathrm{m}$, inertial collision and gravity effect were obvious; when the particle size of dust droplets $d_{w}$ was less than $0.4 \mu \mathrm{m}$, diffusion and electrostatic attraction effect were distinct; when the particle size of dust was in the range $0.4 \mu \mathrm{m}<d_{w}<40 \mu \mathrm{m}$, all the various trapping effect for the droplets should not be neglected (Fig. 3).

In the actual experiment, various trapping effects exist simultaneously, as far as possible to improve the calculation accuracy, and the combined effects of various trapping effect can be calculated as follows [14]:

$$
\eta=1-\left(1-\eta_{G}\right)\left(1-\eta_{I}\right)\left(1-\eta_{R}\right)\left(1-\eta_{S}\right)\left(1-\eta_{E}\right) .
$$

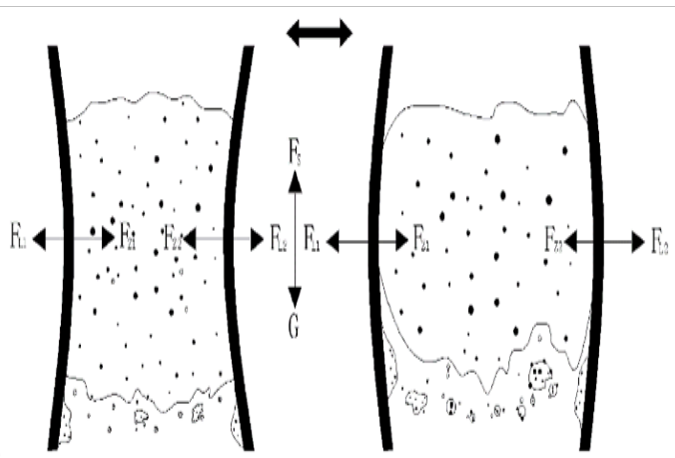

Fig. 4 Force diagram of water film containing dust.

\section{Analysis of dust removal mechanism in vibration fiber grid}

Because of the action of fluid, the dense fiber grid produces self-excited vibration. When the natural frequency of the adjacent filaments is consistent with the vortex shedding frequency, the induced resonance forms a water film between the adjacent fibers. Therefore, the effective cross-sectional area of grating fiber increases, the efficiency of charged droplets contains immense dust, and dust collecting is greatly increased. After the rupture of the water membrane, which carries a large amount of dust, it directly subsides due to its gravitational effect. The periodic formation and rupture of this water membrane are called resonance film-making effect, which plays a role in collecting dust by the vibrating fiber grid. The force diagram of water film containing dust is shown in Fig. 4.

\section{Calculation of vibration transverse amplitude of intensive vibration fiber grid}

Because the fibers in the experiment mainly undergo transverse vibrations, the transverse vibrations of the fibers are only considered. In confined spaces, the resonance response of both the filament and the vortex shedding can be reduced to the forced vibration of elastomer. Because the fiber grid is fixed up and down, under the effect of constant airflow, the characteristics of resonance of membrane filaments can be quantified as the resonance amplitude of vibrating fiber grid [23], and the wave equation is shown as follows:

$$
\left.\begin{array}{rl}
T \frac{\partial y}{\partial z^{2}}+F(2, t) & =\rho_{L} \frac{\partial y}{\partial t} \quad(0<z<L, t>0) \\
y(2,0) & =0 \quad(0<z<L) \\
\frac{\partial y(2,0)}{\partial t} & =0 \quad(0<z<L) \\
y(0, t) & =y(L, t)=0 \quad(t \geqslant 0),
\end{array}\right\}
$$


where $\rho_{L}$ is the density of linear system (including the added mass of fluid), $\mathrm{kg} / \mathrm{m}^{3} ; F(2, t)$ is an external force acting on a filament (including the damping components and fluid forces on an integrated fiber in fiber cross section lateral force $F_{L}$ ), N; and $T$ is the filament tension, $\mathrm{N}$.

Because $F_{L}$ is periodic with time $t$, it can be expressed through trigonometric functions:

$$
\left.\begin{array}{rl}
\frac{F_{L}(2, t)}{\rho_{L}} & =\bar{f}_{L} \cos (k, f), \\
\bar{f}_{L} & =\frac{1}{2} \rho_{S} D L M_{L} U^{2}, \\
f_{S} & =\frac{\operatorname{Sr} U}{D},
\end{array}\right\}
$$

where $\bar{f}_{L}$ is the amplitude of fiber transverse, $\mathrm{m} ; k$ is the angular frequency, $k=2 \pi f ; f$ is the natural frequency; $k_{S}$ is the angular frequency of vortex shedding; $k_{L}$ is the angular frequency of fiber; $f_{S}$ is the natural frequency of vortex shedding; and $f_{L}$ is the natural frequency of fiber.

According to the vibrational condition of the fiber, the Sr number can be used in the form of empirical formula:

$$
\mathrm{Sr}=0.198\left(1-\frac{19.7}{\operatorname{Re}}\right) \quad(\operatorname{Re}<260) .
$$

By separating variables, the orthogonal vibration equation is transformed into Taylor series expansion, and the three powers of single degree of freedom resonance equation are intercepted, and the resonance amplitude is calculated as follows:

$$
Y_{\max } \approx \frac{\bar{f}_{L}}{2 Y k_{L}^{2} m},
$$

where $m$ is the total quality of fibers and fixtures, $\mathrm{kg} ; Y$ is the damping ratio, $Y=C / 2 m k_{L} ; C$ is the damping coefficient of fiber material, $\mathrm{N} \cdot \mathrm{s} / \mathrm{m}$.

When resonance occurs, $f_{S}=f_{L}$, and Eqs. (15) and (16) are substituted into Eq. (17), leading to Eq. (18):

$$
Y_{\max } \approx \frac{\rho_{S} U L D^{2}\left(2 \int_{0}^{2 \pi} \omega \cos \theta \mathrm{d} \theta-\operatorname{Re} \int_{0}^{2 \pi} P \sin \theta \mathrm{d} \theta\right)}{792 \pi c(\operatorname{Re}-19.7)} .
$$

The resonant amplitudes of the dense filaments, which are induced by the constant fluid effect, are related to the following factors:

(i) external condition factor: $\rho_{S}, P, \operatorname{Re}, U$;

(ii) fiber grid physical structure factor: $L, D$ (changes in its size can be equivalent to the change in the fiber gap);

(iii) fiber material factors: $\omega, C$.

\section{Theory of dust collection efficiency by intensive vibration fiber}

By using infinitesimal analysis method, the fiber grating is divided into innumerable thickness for $\mathrm{d} h$ infinitesimal body, and the mass concentration of the element in the micro body is $m_{0}$; therefore, the equivalent total length of the fiber grating is calculated as follows:

$$
L_{a}=\frac{\beta A \mathrm{~d} h}{\pi d^{2}},
$$

where $\beta$ is the total volume filling rate of fiber grating, expressed by the formula

$$
\beta=\frac{\pi N_{1} L d^{2}}{A H},
$$

where $N_{1}$ is the fiber grating number.

Based on the theory of the type (19), the equivalent capture volume of the fiber grid is calculated as follows:

$$
N=2 d U L_{a} m \eta=\frac{2 d U m \eta \beta A d h}{\pi d^{2}},
$$

where $m$ is the mass concentration in fiber gratings, $\mathrm{kg} / \mathrm{m}^{3}$.

Under ideal conditions, the reduced amount of mist is equal to the amount of catcher in the fiber grid fluid within the unit time.

$$
-A U_{0} \mathrm{~d} m=2 m d \eta \frac{U_{0}}{1-\beta} \frac{A \beta \mathrm{d} h}{\pi d^{2}},
$$

where $U_{0}$ is the infinitesimal body speed expressed as $U_{0}=U(1-\beta) ; N_{1}$ is the fiber grating number; $m$ is the mass concentration in fiber gratings, $\mathrm{kg} / \mathrm{m}^{3}$.

Assuming that $C_{1}=(2 \eta / \pi d)[\beta /(1-\beta)]$, Eq. (22) is simplified and is integrated as follows:

$$
\int_{m_{0}}^{m} \frac{\mathrm{d} m}{m}=\int_{0}^{H}-C_{1} \mathrm{~d} h .
$$

After arrangement, the theory capture efficiency by intensive filaments is calculated as follows [12]:

$$
\eta_{a}=1-\frac{m}{m_{0}}=1-\mathrm{e}^{-C_{1} H} .
$$

\section{Determination of the range of experimental corona voltage}

At the same flow rate, when the spray pressure was higher, the spray volume was larger, but the corona voltage was easy to break down. The range of the corona voltage also increased with increasing spray 


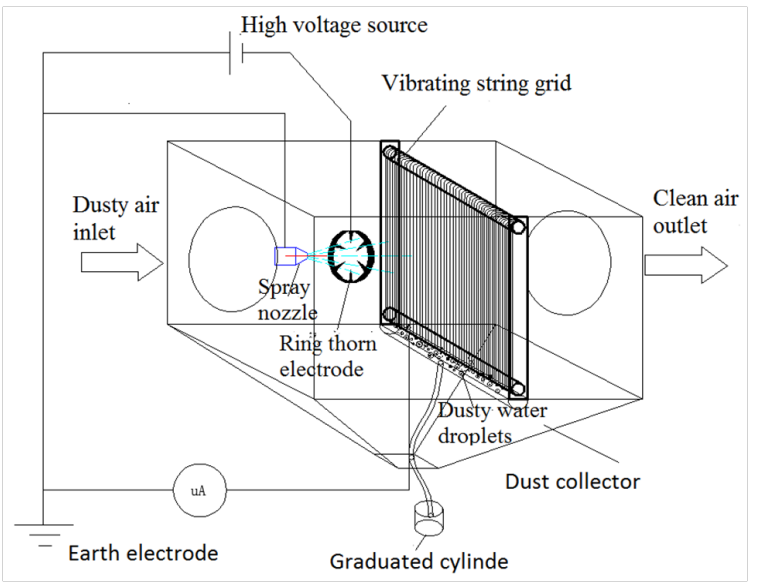

Fig. 5 Determination of voltage-current characteristics and settlement rate of fiber grating.

water pressure, within a certain range $0.7 \mathrm{MPa}$, and the spray water pressure was set to a maximum value $0.5 \mathrm{MPa}$. The inlet wind speed was mainly measured by six wind states. The insulation of high voltage direct current was obtained by the polytetrafluoroethylene body, on which water mist could not be sprayed, achieving the corona discharge. The experimental device was mainly a warp direction fiber grid for a different material in longitudinal direction of fiber grating, as shown in Fig. 5.

In the experiments, the distance between the nozzle and the corona ring was $75 \mathrm{~mm}$, and the spacing between the corona ring and fiber grating was $11 \mathrm{~cm}$. The dust emission rate was $0.2 \mathrm{~g} / \mathrm{s}$ in the dust generator, and the air density $\rho$ was $1.171 \mathrm{~kg} / \mathrm{m}^{3}$. Adjusting to the inlet wind velocity, the average inlet wind speed was $8.6 \mathrm{~m} / \mathrm{s}$. The voltampere characteristic curve was measured for fiber grids of different materials, as shown in Fig. 6.

As shown in Fig. 6ab, the corona voltage and fiber grating corona current curve in the condition with water spraying were basically the same as that of the corona discharge curve without water spraying. The voltage of the test was same as the voltage of the corona region, and the experimental data was valid. The variation in the breakdown voltage of different inlet velocity under other same experimental conditions was less, in the range $0-2 \mathrm{kV}$. When the same voltage was applied, the corona current in the water spray was greater than that without water spraying, and the directional movement of charged particles between the nozzle and the corona electrode was obvious. The difference in charge trapping ability of fiber grids of different materials under non-spray conditions was obvious, with the increase in corona voltage in the following order: polyester $>$ stainless steel $>$ plastic $>$ nylon, in the presence of spray conditions, the difference in charge trapping ability of fiber grids of various materials was not significant, indicating that water mist could improve the efficiency of dust charging and enhance the ability of fiber grids to trap charged dust-containing water mist, thus may reduce the requirements for fiber grids materials.

As shown in Fig. 6b, the breakdown voltages of nylon, plastic, polyester, and stainless steel wires were measured to be $46,41,46$, and $43 \mathrm{kV}$, respectively. The voltage range of four materials was determined according to the size of the electronics corona breakdown: nylon $=$ polyester $>$ stainless steel fiber $>$ plastic fiber, and its corona voltage range was taken as $5-41 \mathrm{kV}$. The other experimental conditions were unchanged, and the experiment was carried out by the plastic fiber gratings, inserted into one, two, three, and four pieces of fiber grid plates, respectively, and the volt-ampere characteristic curve was measured, as shown in Fig. 7a.

According to Fig. 7a, the moving velocity of the charged particles between the corona and fiber grating increased, the corona current also increased at the same voltage and with increasing number of the plastic fiber grating; however, the resistance of the directional flow in the dust collector caused the increment.

The experimental device was a plastic fiber grating. By adjusting the inlet wind velocity, the volt-ampere characteristic curve was obtained when the distance between the nozzle and the corona ring was $75 \mathrm{~mm}$, with the water spray in the electronic corona. The experimental results are shown in Fig. 7b. With increasing inlet wind speed, the efficiency of the fiber grille in the trapped charged particles decreased, thus decreasing the corona current.

Under the condition of water spray, and with the inlet wind velocity $v_{1}=8.12 \mathrm{~m} / \mathrm{s}$, the voltampere characteristic curve of electronic corona was measured at different distances between the nozzle and corona ring $(25,50$, and $75 \mathrm{~mm})$, as shown in Fig. 7c.

As shown in Fig. 7c, the ranges of corona voltage were $5-23 \mathrm{kV}(25 \mathrm{~mm}), 5-32 \mathrm{kV}(50 \mathrm{~mm})$, and 5-36 kV $(75 \mathrm{~mm})$ in different distances between the nozzle and corona ring. The range of corona voltage was obviously affected by the distance between the nozzle and corona ring; when the distance was large, the range of corona voltage was 

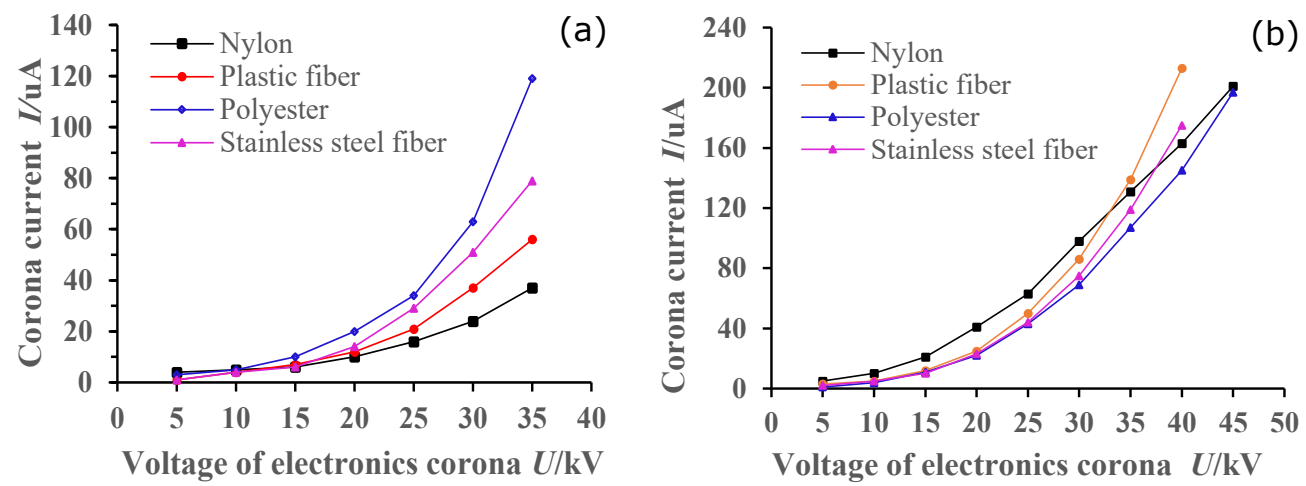

Fig. 6 The volt-ampere characteristics of different materials in (a) without water spray and (b) with water spray.
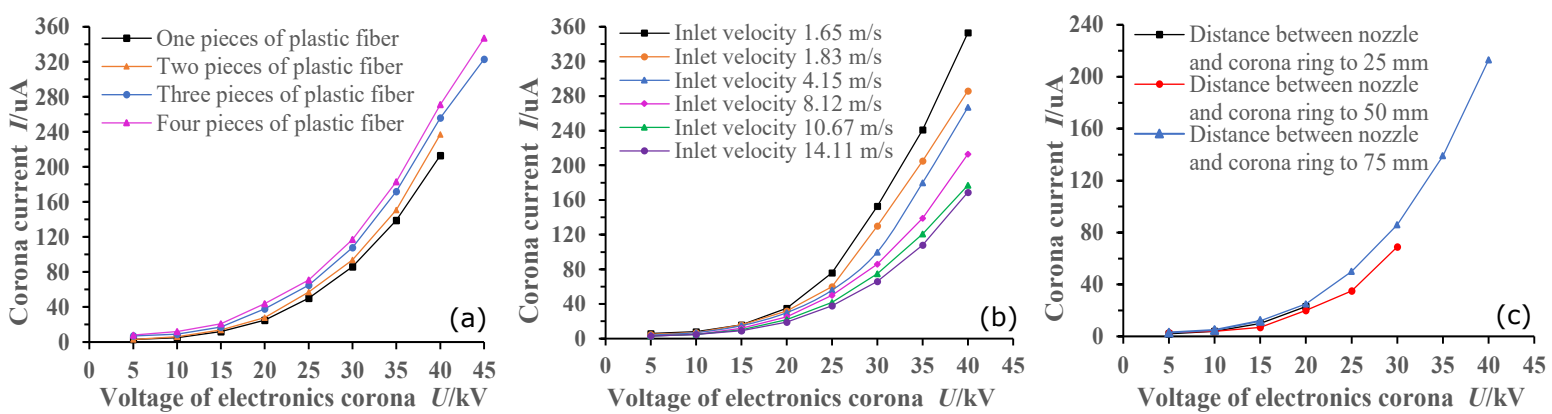

Fig. 7 The volt-ampere characteristic curves of different experimental factors: (a) fiber grating, (b) spray spacing $75 \mathrm{~mm}$ with water spray, and (c) different distances between the nozzle and corona ring.

wider. In the range $5-20 \mathrm{kV}$, the electric corona current first decreased under the same voltage, and then increased with increasing distance between the nozzle and corona ring. Therefore, the optimum spray interval was selected in order to obtain the optimum electric corona effect. Fig. 7c shows that the distance between the nozzle and the corona ring was $75 \mathrm{~mm}$, which was chosen as the experimental spray spacing with the optimum corona charging effect, and the range of corona voltage was large. The range of corona voltage was determined as 5$34 \mathrm{kV}$ in the experiment.

\section{Resistance loss and efficiency of dust collection by longitude of fiber grating, latitude, and interlacing fiber grating}

In the experiment, the pressure of spray water was set as $0.5 \mathrm{MPa}$, and the corona voltage was $30 \mathrm{kV}$. A piece of fiberboard was inserted, and the distance between the corona ring and fiber grating was $11 \mathrm{~cm}$. The dust emission rate was $0.2 \mathrm{~g} / \mathrm{s}$ in the dust generator, and the air density $\rho$ was $1.165 \mathrm{~kg} / \mathrm{m}^{3}$.

The resistance losses to the fiber grating with longitude, latitude, and interlacing arrangement were measured, and the inlet wind speed is shown in Fig. 8a. The efficiencies of dust collection were measured by longitude of fiber grating, latitude, and interlacing fiber grating, and the results are shown in Fig. 8b.

Fig. 8a shows that with increasing wind speed, the resistance loss of three types of fiber gratings increased, namely in the order interlacing fiber grating $>$ latitude $>$ longitude of fiber grating, but the resistance loss of interlacing fiber grating was the greatest.

Fig. 8b shows that the efficiency of dust collection by longitude of fiber grating first increased and then decreased with increasing inlet wind velocity. The efficiency of dust collection by other two fiber arrangement decreased with increasing inlet wind velocity. The efficiency of dust collection by longitude of fiber grating and filling warp blends were more than $80 \%$, and the difference between the two was small. The efficiency of dust collection by latitude of fiber grating was always less than those by the former two, and fell below $80 \%$ with further increase in the wind speed.

The droplets with dust in the longitude of fiber grating fell down because of the gravity of the dust 

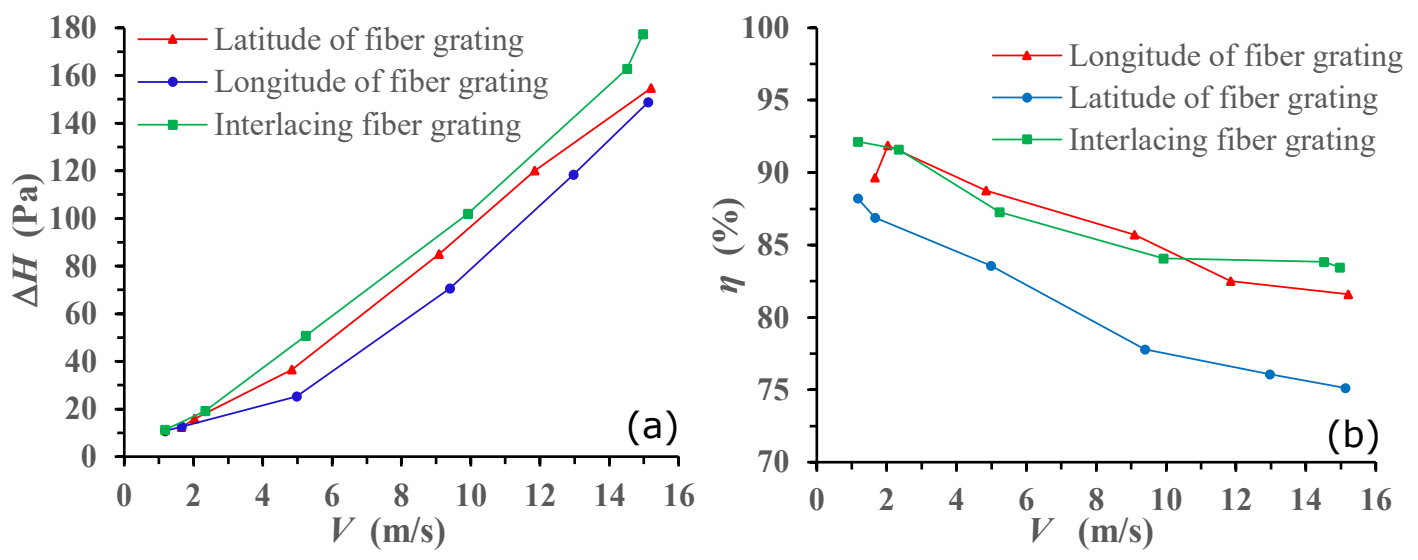

Fig. 8 (a) Resistance loss and (b) efficiency of dust collection of various fiber arrangements.

droplets in the experiment. The dust and water droplets were less remained on the fiber filaments, which can be used continuously. There were more dust droplets in the latitude of fiber grating, and the residual droplets condensed and hanged on the filaments. As a result, the gap between the fibers shrank and became uneven, and the efficiency of dust removal declined sharply. The latitude of fiber grating should be taken out, cleaned, and dried before reuse. There was a lot of dust and droplets in the interlacing fiber grating, and many of the pores clogged with dust. The increase in the resistance of the fiber grating was obvious, and the interlacing fiber grating should be taken frequently out, cleaned, and dried.

Combined with the dust removal efficiency and the size of the resistance loss in various types of fiber grating, the longitudinal fiber grating is better than the other two in terms of dust removal and resistance loss. Therefore, the longitudinal fiber grating was used in this experiment.

\section{DISCUSSION}

Dust removal efficiency and resistance loss are two important indicators for evaluating the performance of dust collectors. The excellent performance of the dust collector is manifested in the efficient dust removal rate and low resistance loss. Gong et al [3] studied the effect of wind speed, water volume, and the number of vibrating wire filter plates on the dust removal efficiency and resistance of a wet vibrating wire dust collector. Under the condition of a certain amount of water spray, the dust removal efficiency and resistance increase with increasing wind speed, but the wind speed is too high, causing the fan to carry water and thus reducing the efficiency of dust removal. Wang et al [24] studied the effect of the filter wind speed on the defogging rate and pressure loss of the vibrating wire grid. He believed that the charged defogging rate was higher than that of uncharged rate, and the pressure loss increases rapidly with increasing filter wind speed. In this study, after determining the corona voltage range of multiple factors that affect the dust removal efficiency of the vibrating wire grid, the optimal value of each affecting factor was selected, and the effect of pressure loss and dust removal efficiency with wind speed under different layouts of the vibrating wire grid was measured. The comprehensive analysis in Fig. 6ab shows that polyester fiber grids have the best charge trapping ability under no spray conditions, and the charge trapping ability of fiber grids of various materials improved under spray conditions, and the difference in charge trapping ability of fiber grids of various materials was not significant, reducing the requirement for fiber grids materials. The analysis in Fig. 7a-c shows that increasing the number of plastic fiber grids could increase the range of corona voltage, increasing the inlet air speed will reduce the corona current, which is not conducive to dust purification in the dust collector, by experimenting with different distances between the nozzle and the plastic fiber grids, it could be seen that the greater the distance between the nozzle and the corona ring, the wider the range of corona voltage, and the best distance between the nozzle and the corona ring was determined as $75 \mathrm{~mm}$. The pressure loss increase with increasing wind speed, and the dust removal efficiency decreases with increasing wind speed. The experimental results were reliable and found to be very consistent with the literature data. The comprehensive analysis in Fig. 8ab shows that when the wind speed is small, the resistance loss of the charged water mist vibrating grid is small, and 
the dust removal efficiency at this time was very high, reaching more than $90 \%$. The dust collector was better at low wind speed performance.

In summary, the charged water mist in the vibrating fiber web overcomes the disadvantages of electrostatic dust removal and vibrating fiber web dust removal. The charged water mist in the vibrating fiber grid has high dust removal efficiency, low resistance, simple structure, and good application prospects.

\section{CONCLUSION}

The five types of dust catching effects, such as gravitational settlement, inertial collision, interception, diffusion, and electrostatic attraction in a single fiber were theoretically analyzed, and the calculation formula of comprehensive efficiency was derived. Moreover, the mechanism of vibration fiber grid, whose resonant film-forming effect was analyzed by spray water, was theoretically studied. Some factors affecting the response amplitude of resonance were investigated when the Reynolds number Re was less than 260.

The efficiency of collection dust and resistance loss in longitude of fiber grating, latitude, and interlacing fiber grating was determined by lots of experiments, in order to determine the fiber grating arrangement. The experiments showed that the dust removal was stable and efficient, the resistance loss was relatively small, and it was a natural dust drop, the fiber structure was stable, and the dust could be automatically cleared by vibration in the longitude of fiber grating, it was easy to use. Therefore, the optimal choice was the longitudinal fiber grating. In addition, the structural parameters and fiber materials were presented in the longitudinal fiber grating in this study, and the results of the fiber grating were demonstrated.

Acknowledgements: This study was supported by the National Natural Science Foundation of China (51864016 and 51464016).

\section{REFERENCES}

1. Deng JW, Qu HW (2017) Studies on motion characteristics of droplet in spray process of WESP. $J$ North China Electr Power Univ 44, 105-110.

2. Steiner D, Lanzerstorfer C (2018) Investigation of dust resistivity for a fractioned biomass fly ash sample during poor combustion conditions with regard to electrostatic precipitation. Fuel 227, 59-66.

3. Gong LH, Liu K, Wang YM (2001) A research on the vibration properties of the single fibers in vibration fiber grid dust collector. $J$ Saf Environ 1, 15-18.
4. Gan CH, Zheng CH, Yang ZD, Gao X (2016). Experimental study of honeycomb experimental-scale wet electrostatic precipitator removing coal-fired fine particulate matter. Acta Sci Circumstantiae 37, 2369-2374.

5. Oliveira AE, Guerra VG (2018) Influence of particle concentration and residence time on the efficiency of nanoparticulate collection by electrostatic precipitation. J Electrost 96, 1-9.

6. Chen ZY, Chen L, Wu CF (2016) Performance of discharge in two phase mixture of air-droplet. Nat Environ Pollut Technol 15, 343-348.

7. Chen ZY, Lai ZH, Wu CF (2016) Study on mode and mechanism of the electrostatic spray charged. $J$ Mech Eng Res Dev 39, 157-163.

8. Chen ZY, Bo J, Wu CF (2014) Research on electric field strength of the longitudinal transverse collecting plates in electrostatic precipitator. BioTechnol Indian $J$ 10, 5700-5710.

9. Huo YF, Wu LY (2017) Industrial application and the configuration of the corona discharge device for moisture removal. Sulphuric Acid lnd 3, 52-56.

10. Luo F, Hu B, Wu H, Yang LJ (2017) Experimental study on removal properties of PM2.5 and sulfuric acid mist by wet electrostatic precipitator. $J$ Southeast University 47, 91-97. [in Chinese]

11. Cooke R, Wilson A (2007) A probabilistic characterization of the relationship between fine particulate matter and mortality: elicitation of European experts. Environ Sci Technol 41, 6598-6605.

12. Natale FD, Manna L, Motta FL, Colicchio R (2018) Capture of bacterial bioaerosol with a wet electrostatic scrubber. $J$ Electrost 93, 58-68.

13. Ding QQ, Dong BY, Chen ZY (2015) Numerical simulation of charged particle and electric field in electrical area of electrostatic filter bag. Nonferrous Met Sci Eng 6, 111-115.

14. Yu YP, Sun XX, Zou LL, Zhang HB, Liu YQ, Liu M (2020) Polycyclic aromatic hydrocarbons (PAHs) in surface soil from the Guan River Estuary in China: Contamination, source apportionment and healthrisk assessment. ScienceAsia 46, 80-86.

15. Chen ZY, Jin B, Wu CF (2012) Application of support vector machine in evaluation of environment air quality. Environ Sci Technol 35, 395-398.

16. Mi JF, Pan Y, Du SN, Li XL (2016) SO2 removing by earthed atomizing corona discharge technology. Chem Eng 44, 6-10.

17. Chen ZY, Zou M, Xiao ZX (2018) Mechanism research of electrically discharged water mist in vibrating fiber filter. Paper Asia Compendium 3, 131-135.

18. Gouëllo M, Hokkinen J, Kärkelä T, Rantanen P, Auvinen A (2018) Development and qualification of an innovative wet electrostatic precipitator in view of gaseous iodine filtration on laboratory-scale. $\mathrm{Nucl}$ Eng Des 327, 7-21.

19. Xiang XD (2002) Modern Dust Removal Theory 
andTechnology, Metallurgical Industry Press, Beijing.

20. Xiang XD, Colbeck I (1997) Charged water drops and smoke dissipation. Fire Saf J 28, 227-232.

21. Ko GH, Ryou HS (2005) Droplet collision processes in an inter spray impingement system. $J$ Aerosol Sci 35, 1300-1321.

22. Jaworek A, Marchewicz A, Sobczyk AT, Krupa A, Czech T (2018) Two-stage electrostatic precipitators for the reduction of PM2.5 particle emission. Prog
Energy Combust Sci 67, 206-233.

23. Yang WG (2019) Eigenvalue problems for nonlinear conformable fractional differential equations with multi-point boundary conditions. ScienceAsia 45, 597-602.

24. Wang Y, Xiang XD, Yao GY (2014) Research on the defogging performance of bipolar corona vibrating grid. Environ Eng 10, 145-147. 